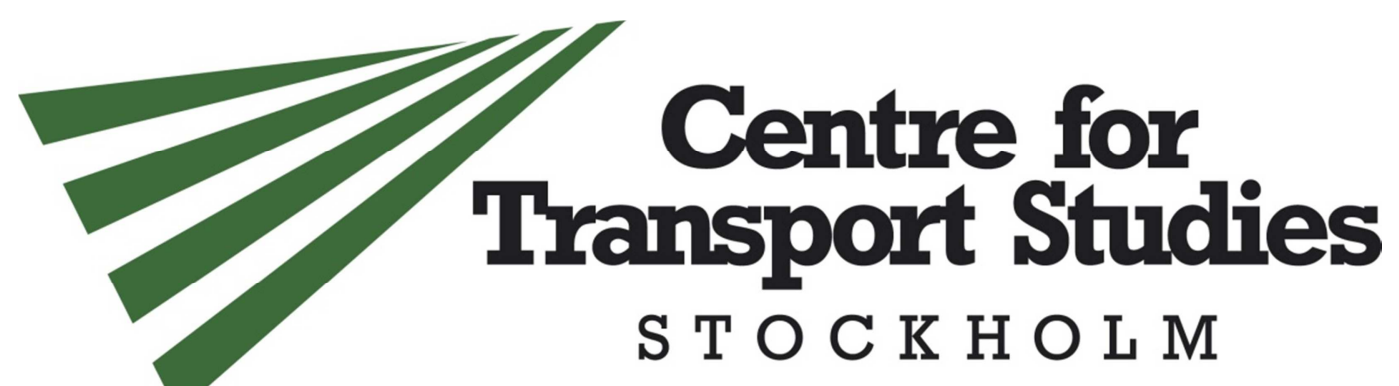

\title{
Valuations of travel time variability in scheduling versus mean-variance models
}

\author{
Maria Börjesson, Royal Institute of Technology \\ Jonas Eliasson, Royal Institute of Technology \\ Joel Franklin, Royal Institute of Technology
}

CTS Working Paper 2012:2

Forthcoming in Transportation Research B (2012)

\begin{abstract}
The standard method of estimating the value of travel time variability for use in policy appraisal is to estimate the parameters of a reduced-form utility function, where some measure of travel time variability (such as the standard deviation) is included. A problem with this approach is that the obtained valuation will in general depend on the standardized travel time distribution, and hence cannot be transferred from one context to another. A recently suggested remedy of this problem has been to estimate a scheduling model, which in theory is transferrable, and use the implied reduced-form to derive valuations for use in appraisal. In this paper we estimate both a scheduling model and the implied reduced-form model, using stated choice data. The valuation of travel time variability implied by the scheduling model turns out to be substantially smaller than what is obtained from a reduced-form model estimated on the same sample. The results suggest that the scheduling model does not capture all of the disutility arising from travel time variability. Hence, although it can be shown that scheduling and reduced-form models are "theoretically equivalent", that hypothesized equivalence is not reflected in the empirical evidence. We speculate that the derivation of reduced-form models from an underlying scheduling model omits two essential features: first, the notion of an exogenously fixed "preferred arrival time" neglects the fact that most activities can be rescheduled given full information about the travel times in advance, and second, disutility may be derived from uncertainty as such, in the form of anxiety, decisions costs or costs for having contingency plans. We also report our estimates of the valuation of travel time variability for public transit trips, for use in applied appraisal.
\end{abstract}

Keywords: Travel time variability, reliability, delays, scheduling models.

JEL Codes: R49, D61

Centre for Transport Studies

SE-100 44 Stockholm

Sweden

www.cts.kth.se
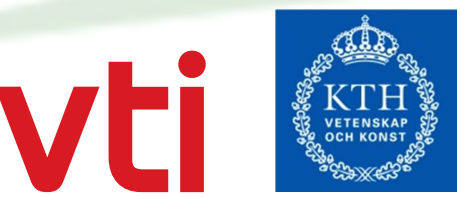


\section{INTRODUCTION}

In recent years, the reliability of transport systems has attracted increasing attention both from researchers and policy-makers. A rapidly growing body of research literature addresses measurement and valuation of travel time variability, and the goal of enhancing reliability seems to take an increasing priority for policy-makers. For example, the Netherlands has introduced reliability as a goal for its transport policies, and the Swedish Government recently declared that more efforts and resources need to be spent on reliability, at the expense of funding for new investments. The importance of reliability is perhaps most apparent in the public transit system: it is now standard that transit operators regularly publish statistics on reliability.

In the analysis of travel time reliability, there are two principally distinct approaches: first, the "scheduling approach", where the traveller's departure and arrival time preferences are made explicit in the model; and second, what may be described as the "reduced-form approach", where some statistical measure of the travel time variability is introduced directly in a reduced-form indirect utility function. While a scheduling approach is often the natural choice for forecasting purposes (e.g. modelling departure time choices), the reduced-form approach is usually the only feasible alternative for appraisal and evaluation purposes. As a measure of the variability in the reduced-form function, most studies have used either the standard deviation of the travel time or the average delay relative to scheduled arrival time, although some studies include both (Bates, Polak, Jones, \& Cook, 2001; Batley, 2007), while some studies use percentiles of the travel time distribution (Lam \& Small, 2001). When the standard deviation is used as the variability measure, the approach is usually termed the "mean-variance" approach.

Reduced-form approaches can be derived from an underlying scheduling model, assuming that travel times follow a known random distribution and that travellers choose their departure time optimally to account for this randomness. This connection between the two approaches has provided a theoretical underpinning for the use of reduced-form approaches in applied appraisal. However, the reduced-form approach has two potentially important drawbacks. First and foremost, an estimated valuation from a reduced-form model will in general depend on the specific travel time distribution - not just on its general properties such as its standard deviation ${ }^{1}$. This means that a "value of standard deviation" obtained in one circumstance, using a particular distribution, cannot easily be transferred to another context where the standardized travel time distribution has a different shape. This relationship has been properly characterized only recently, in Fosgerau and Karlström (2010), even if the insight had been pointed out earlier (Bates, Black, Fearon, Gilliam, \& Porter, 2002). Second, estimating reduced-form models rests on the possibility to present travel time distributions to respondents in a stated choice experiment, and then having them indicate which they would prefer. The problem of presenting travel time distributions comprehensibly to respondents has proven difficult - especially since the valuation will depend on the shape of the tail of the distribution, so it is important to convey enough information about the "far end" of the distribution to the respondent.

\footnotetext{
${ }^{1}$ However, there are some scheduling models that result in reduced-form models that are independent of the standardized travel time distribution. The Vickrey (1973) model analyzed by Fosgerau and Engelson (2010) and used in this paper is an example of this.
} 
These two arguments have recently led researchers to discuss an alternative to the current practice of how valuations of travel time variability are estimated. Rather than estimating marginal valuations in a reduced-form expression directly, one should perhaps estimate a scheduling model first, and then derive a reduced-form valuation that can be used for appraisal, given the relevant travel time distribution in each applied context (see Bates (2009) for a review of these discussions). This solves, in principle, the two problems with the reduced-form approach - its dependence on the specific travel time distribution, and the need to present travel time distributions to respondents - while maintaining its main strength: that one does not need to model travellers' choices explicitly, nor use information about the distribution of preferred arrival times. The possibility to explicitly derive a reduced-form approach from a scheduling model for any given travel time distribution was made possible by a groundbreaking paper by Fosgerau and Karlström (2010). This was followed up by Fosgerau and Engelson (2011), who give an analogous derivation using a different scheduling model.

To be sure, this potential equivalence between the reduced-form and scheduling approaches rests on some strong assumptions. Most importantly, it assumes that travellers optimize their travel choices so as to maximize utility, and that under uncertainty they have accurate perceptions of the probability distributions. Some important contributions to econometrics have identified deviations from such utilitymaximizing behaviour: for example, a substantial body of research has found systematic misperceptions of risk levels, and in particular that very small risks are overweighed (Lichtenstein,(1978); Morgan et al., (1983); Hakes and Viscusi, (2004); Burns et al., (2010)). However, findings so far in travel behaviour have shown that such misperceptions are likely to be less of a problem when travellers have gained substantial experience from similar trips (de Palma et al., 2008). Since this paper focuses on daily commute travel behaviour, one can reasonably assume that most travellers have a substantial amount of experience making the same trip.

The main focus of this paper is on testing the transferability between the scheduling and reduced form approaches. We conduct such a test on two alternative specifications of scheduling preferences, one with a step function for marginal utilities and another with sloped marginal utilities. We have collected stated choice (SC) data from respondents making a public transit trip. Respondents participated in two different binary choice experiments relating to the observed trip, one for estimating a scheduling model and one for estimating a reduced-form model. Theoretically, the two models should be possible to "translate" into each other: the estimated parameters of the scheduling model imply specific values of a reduced-form function, which should then, in principle, coincide with the parameters estimated directly in the second stated choice experiment. If that is indeed the case, then this opens up a way to circumvent the problems with the reduced-form approach. On the other hand, if the two estimated models are not consistent, then this means that one of the assumptions underlying the equivalence between these two approaches is flawed. For example, something could be missing from the underlying derivation of the reduced-form expression, and in that case one needs to understand why and how our theory deviates from how travellers actually make travel choices.

The possibility that scheduling models might not be able to capture the entire disutility of travel time uncertainty has been observed before. Noland et al. (1998) hypothesize that the pure nuisance of not being able to plan activities precisely could, in addition to 
scheduling costs, play a role in understanding aversion to travel time uncertainty. They denote this possible additional cost a "planning cost". However, they do not investigate the precise relationship between "scheduling" and "uncertainty" costs, primarily because the necessary theoretical framework was not available at the time.

In order to test the consistency of the scheduling and reduced form models, we consider two different scheduling models: one that we call the "step" model since the marginal utility of being at the destination has a "step" at the preferred arrival time, and another that we call the "slope" model since the marginal utilities of being at the origin and destination, respectively, change linearly over time. These two scheduling models imply different reduced-form expressions: the "step" model results in an indirect utility function containing the standard deviation of travel time (as shown by Fosgerau and Karlström, 2010), while the "slope" model gives an indirect utility function containing the travel time variance (as shown by Fosgerau and Engelson, 2011).

Beyond the theoretical equivalence of the scheduling and reduced-form models, our findings also have implications for putting new practices into perspective. Historically, different measures of travel time variability were introduced for appraisal purposes in a rather ad-hoc manner, without any proper theoretical motivation. The current theoretical motivation, that variability measures such as standard deviation or variance can be formally derived from scheduling models, is a fairly recent insight ${ }^{2}$. However, to our knowledge there have been no investigations into whether the theoretical equivalence between the two approaches holds empirically, i.e. whether the valuation implied by a certain scheduling model coincides with the valuation obtained from the corresponding reduced-form model. If not, then this is a signal that one of the models captures something that the other does not, and it may be important to understand what this might be.

The paper is organized as follows. Section 2 is devoted to explaining the relation between the scheduling and reduced-form approaches more precisely, hence making the research question more precise. In section 3 , we present the survey data. The main estimation results are presented in section 4, followed by a discussion of their implications in section 5 . Section 6 concludes.

\section{THEORY}

The setting of this work is the choice of departure time by travellers with a specific origin, destination, general time of day, and mode of travel. We conceptualize that travellers choose their departure times such that they maximize a utility function that reflects considerations such as lost time at the origin, lost time at the destination, the burden itself of time spent en route, and monetary costs. In cases where the travellers face uncertain travel times, we assume that they choose departure time by maximizing expected total utility.

\footnotetext{
2 The manner in which theory has trailed application here is rather similar to how the widely used "gravity models" were theoretically motivated only after a long period of practical application, through the work by Wilson $(1967,1970)$, Snickars and Weibull (1977) and several others.
} 
The notion that the value of variability in travel times can be tied to scheduling decisions is an extension of the idea that the value of a travel time saving derives largely from the opportunity cost of foregoing other activities. Vickrey (1973) expressed this idea in terms of flexible functions for the marginal utility of time spent at the origin and at the destination, respectively. The idea was re-introduced and elaborated by Tseng and Verhoef (2008), and subsequently applied by Jenelius et al. (2011).

Denote the marginal utilities of being at the origin and at the destination, relative to time spent travelling, with $h(x)$ and $w(x)$, respectively, where $x$ is clock time. Without loss of generality the marginal value of time spent travelling is normalized to zero. Then, we can express total utility as the accumulated utility of time spent at the origin and, subsequently, time spent at the destination as:

$U(d, a)=\int_{A}^{d} h(x) d x+\int_{a}^{B} w(x) d x$

Here, $A$ and $B$ are arbitrary beginning and end times of the analysis time period of interest (spanning the range of possible departure and arrival times), and $d$ and $a$ are the respective times of departure and arrival. In the following, we normalize the total utility level by taking the relative utility $u(d, a)=U(d, a)-U(0,0)$, where $U(0,0)=$ $\int_{A}^{0} h(x) d x+\int_{0}^{B} w(x) d x$. This way we can evaluate $u$ in terms of bounds at zero, rather than at $A$ and $B: u(d, a)=-\int_{d}^{0} h(x) d x-\int_{0}^{a} w(x) d x$. In particular, $u(0,0)=$ $-\int_{0}^{0} h(x) d x-\int_{0}^{0} w(x) d x=0$. What form the functions $h(x)$ and $w(x)$ take turns out to be critical for the development of the analysis. To motivate taking a trip, it must at least be the case that at some point in time $w(x)$ exceeds $h(x)$. Both $w(x)$ and $h(x)$ must be positive to make the value of a travel time saving positive.

In our analysis, we take two alternative specifications of the general form given in (1). First, Tseng and Verhoef (2008) illustrated that the general form above can be restricted to give rise to the well-known scheduling model that Small (1982) defined, based on earlier work by Vickrey (1969). This constitutes what we term the "Step Model" due to the step-function taken on by the marginal utility of time spent at the destination. Section 2.1 describes the Step Model, and derives the corresponding reduced-form expression (i.e. the maximal total expected utility, assuming optimal choice of departure time). This expression turns out to be a weighted sum of the expected travel time and an expression including the standard deviation of the travel time. The second specification we use is termed the "Slope Model", and is based on Fosgerau and Engelson (2011) and Vickrey (1973), who take the functions $h(x)$ and $w(x)$ to be linear functions of clock time. Section 2.2 describes the Slope Model, and derives the corresponding reduced-form expression. This expression turns out to be a weighted sum of the expected travel time, its square, and the variance of the travel time.

\subsection{The "Step Model"}

The first scheduling model draws from Vickrey (1969) and Small (1982), as adapted by Fosgerau and Karlström (2010) and Tseng and Verhoef (2008). Assume that the functions $h(x)$ and $w(x)$ are as follows:

$$
\begin{gathered}
h(x)=\alpha \\
w(x)= \begin{cases}\alpha-\beta & x \leq 0 \\
\alpha+\gamma & x>0\end{cases} \\
5
\end{gathered}
$$


The total utility becomes the well-known Vickrey-Small scheduling model:

$u(d, a)=-\alpha(a-d)+\beta \min (0, a)-\gamma \max (0, a)$.

The specification rests on the assumption that there is a specific point in time when the marginal utility of being at the destination suddenly increases (presumably because some scheduled activity starts then). This point is often called the "preferred arrival time" (PAT). Without loss of generality, we can normalize PAT to $x=0$ as in (2). We refer to this model as the "step" model due to the shape of $w(x)$.

The marginal utilities in (2) can be interpreted in comparison to time spent elsewhere: $\alpha>0$ for time spent at the origin rather than travelling, $\beta>0$ for time spent at the destination before the PAT rather than spent at the origin, and $\gamma>0$ for time spent at the destination after the PAT rather than spent at the origin. For consistency, it is necessary that all parameters are positive and that $\beta<\alpha$. This will give the following order of the marginal utilities: at the destination after the PAT $(\alpha+\gamma)>$ being at home $(\alpha)>$ at the destination before the PAT $(\alpha-\beta)>$ travelling $(0)$. The step model is represented graphically in Figure 1 . The shaded areas represent the total utility function (2), i.e. the integral of the marginal utilities.

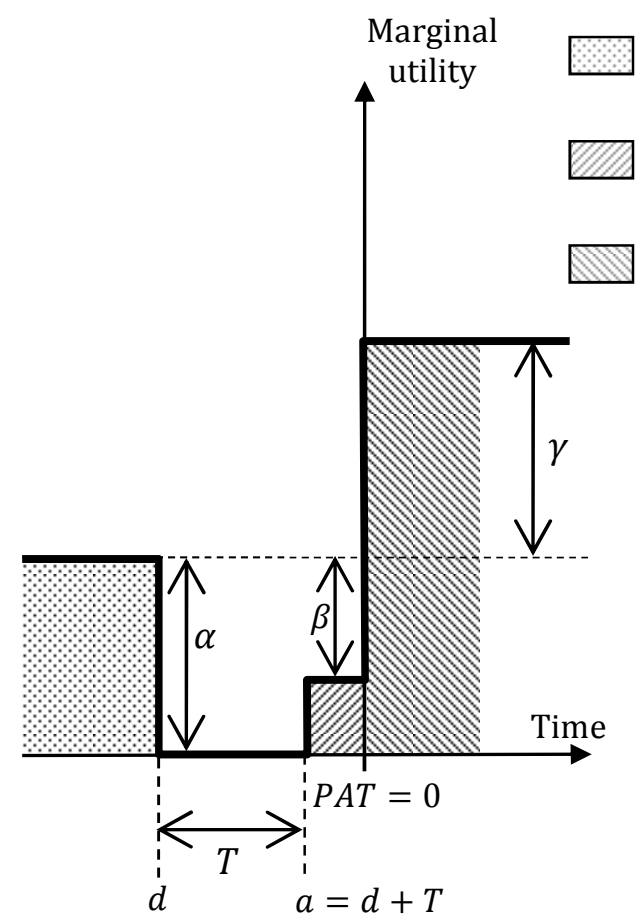

(a) Early Arrival
Utility of time spent at the Marginal origin utility

Utility of time spent at the destination before PAT

Utility of time spent at the destination after PAT

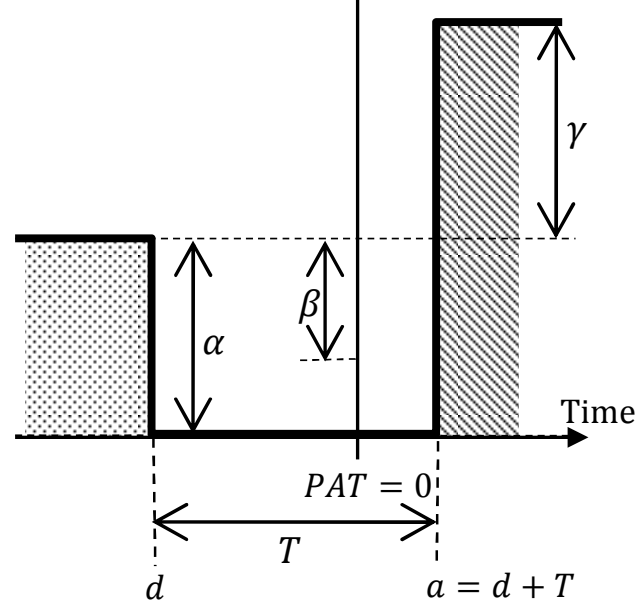

(b) Late Arrival

Figure 1. Marginal utilities of the Step Model. The shaded areas show the total utility as defined in (1) and (2).

\section{Reduced Form}

Now, assume that the travel time $T$ is random, distributed as $T \sim \mu+\sigma X$, where $X$ is a standardized random variable with mean 0 , standard deviation 1 , density $\phi$ and cumulative distribution $\Phi$. If the traveller can choose departure time $d$ freely and the 
distribution of $T$ is independent of $d$, then it can be shown (Fosgerau and Karlström, 2010) that the optimal departure time $d^{*}$ is:

$d^{*}=\underset{d}{\operatorname{argmax}}[E\{-\alpha(a-d)+\beta \min (0, a)-\gamma \max (0, a)\}]=-\mu-\sigma \Phi^{-1}\left(1-\frac{\beta}{\beta+\gamma}\right)$

The maximal expected total utility becomes:

$$
\begin{aligned}
& u^{*}=\max _{d}[E\{-\alpha(a-d)+\beta \min (0, a)-\gamma \max (0, a)\}] \\
& =-\alpha \mu-(\beta+\gamma) \sigma \int_{1-\frac{\beta}{\beta+\gamma}}^{1} \Phi^{-1}(s) d s \\
& =-\alpha \mu-\left\{(\beta+\gamma) H\left(\Phi, \frac{\beta}{\beta+\gamma}\right)\right\} \sigma
\end{aligned}
$$

The last formula is the reduced-form expression corresponding to the "step" scheduling utility function: it is the maximal utility achieved assuming optimal choice of departure time. Note that this is the commonly used "mean-variance" expression, where the utility depends on a weighted sum of the mean and standard deviation of the travel time. But the reduced-form expression also depends on $H$, a functional depending not only on the parameters $\beta$ and $\gamma$, but also on the standardized distribution $\Phi$ - more specifically, the shape of the tail of $\Phi^{-1}$ beyond $1-\frac{\beta}{\beta+\gamma}$. The quantity $H$ can be termed the "mean lateness factor", since it is the mean lateness conditional upon the traveller arriving after the PAT.

In the second stated choice experiment, we will use a particularly simple travel time distribution, namely the binary distribution where the travel time is $t$ with probability $1-p$ and $t+L$ with probability $p$. In other words, there is a delay of length $L$ with probability $p$. In this case, the reduced-form expression (4) becomes ${ }^{3}$ (see Appendix A):

$u^{*}=\left\{\begin{array}{ccc}-\alpha t-[(\alpha+\gamma) p] L & \text { if } \frac{\beta}{\beta+\gamma} \geq p & (\text { Case I) } \\ -\alpha t-[\beta+(\alpha-\beta) p] L & \text { if } \frac{\beta}{\beta+\gamma} \leq p & \text { (Case II) }\end{array}\right.$.

For any particular individual facing a particular risk of delay, we see one of these two cases depending on whether $\frac{\beta}{\beta+\gamma} \leq p$. In Case I, an individual with a low cost of arriving late, relative to arriving early, will maximize utility by choosing a later departure, while in Case II, an individual with relatively high cost of arriving late will optimize by choosing an earlier departure. For a population, there is likely to be a mixture of relative utilities, resulting in a mixture of Cases I and II.

The first stated choice experiment involves deterministic departure and arrival times, and from this we will estimate the parameters $\alpha, \beta$ and $\gamma$ in the scheduling model. In the second stated choice experiment, travel times are random (since delays occur with some probability), and from this we will estimate a reduced-form expression with a set of parameters $\theta$ on the form

\footnotetext{
${ }^{3}$ Note that while Fosgerau \& Karlström (2010) assume a continuous distribution, the same result emerges from the discrete distribution we have chosen. We show this in Appendix A, and a general result has been shown by Hjorth (2010).
} 
$u=-\theta_{1} t-\theta_{2} p L$

If the step model correctly describes travellers' choices, then we should get $\theta_{1}=\alpha$ and either $\theta_{2}=\alpha+\gamma$ if $\frac{\beta}{\beta+\gamma} \geq p$ or $\theta_{2}=\alpha+\beta\left(\frac{1}{p}-1\right)$ if $\frac{\beta}{\beta+\gamma} \leq p$. Evidently, the scheduling model predicts that the $\theta_{2}$ parameter should vary with $p$. We will therefore estimate separate $\theta_{2}$ variables for the four different risk levels $p_{1}-p_{4}$ in the experiment:

$u=-\theta_{1} t-\left(\theta_{2}^{p_{1}}+\theta_{2}^{p_{2}}+\theta_{2}^{p_{3}}+\theta_{2}^{p_{4}}\right) p L$

The parameters $\theta_{2}^{p_{1}}-\theta_{2}^{p_{4}}$ capture a mixed effect of the two parts of (5), depending on the relative portions of the population that fall under each case. It can be shown that the $\theta_{2}$ parameters will be lower for higher risk levels (Börjesson \& Eliasson, 2011). This means that if the step model correctly describes travellers' choices, estimation results from the two choice experiments should give

$\alpha=\theta_{1}$ and $\alpha+\gamma=\theta_{2}^{p_{1}}$

assuming that $p_{1}$ is sufficiently small so $\frac{\beta}{\beta+\gamma} \geq p_{1}$ holds. The first condition implies that the value of travel time savings should coincide in the two stated choice experiments. The second condition implies that the value of expected delay should coincide in the two experiments.

\subsection{The "Slope Model"}

Another specification of the general scheduling form, proposed by Vickrey (1973) and Tseng and Verhoef (2008) and analysed by Fosgerau and Engelson (2011), is to assume that the marginal utilities $h(x)$ and $w(x)$ change linearly with time $x$ :

$h(x)=\beta_{0}+\beta_{1} x$

$w(x)=\beta_{0}+\gamma_{1} x$

As before, the marginal utility of time spent travelling is assumed to be constant and normalized to zero. Without loss of generality, we can assume that $h(x)$ and $w(x)$ intersect at time $0 ; \beta_{0}$ is the common intercept at time $0 . \beta_{1}$ is the rate of change of the marginal utility of spending time at the origin, and $\gamma_{1}$ is the rate of change of the marginal utility of spending time at the destination. For a trip to occur, we need only that $\gamma_{1}>\beta_{1}$; though it is natural to expect their signs to be opposites, $\gamma_{1}>0>\beta_{1}$. We refer to this model as the "slope" model. The model is represented in Figure 2, where the total utility is shown in the shaded areas. The relative utility given departure time $d$ and arrival time $a$ is:

$u(d, a)=-\int_{d}^{0}\left(\beta_{0}+\beta_{1} s\right) d s-\int_{0}^{a}\left(\beta_{0}+\gamma_{1} s\right) d s=$
$-\beta_{0}(a-d)-\frac{\gamma_{1}}{2} a^{2}+\frac{\beta_{1}}{2} d^{2}$.

\footnotetext{
${ }^{4}$ This result is equivalent to the result from Fosgerau and Engelson (2010) except that they take the additional normalization that $\beta_{1}=\gamma_{1}-1$ and they measure utility in reference to an arbitrary early departure time and late arrival time, rather than to an ideal trip.
} 


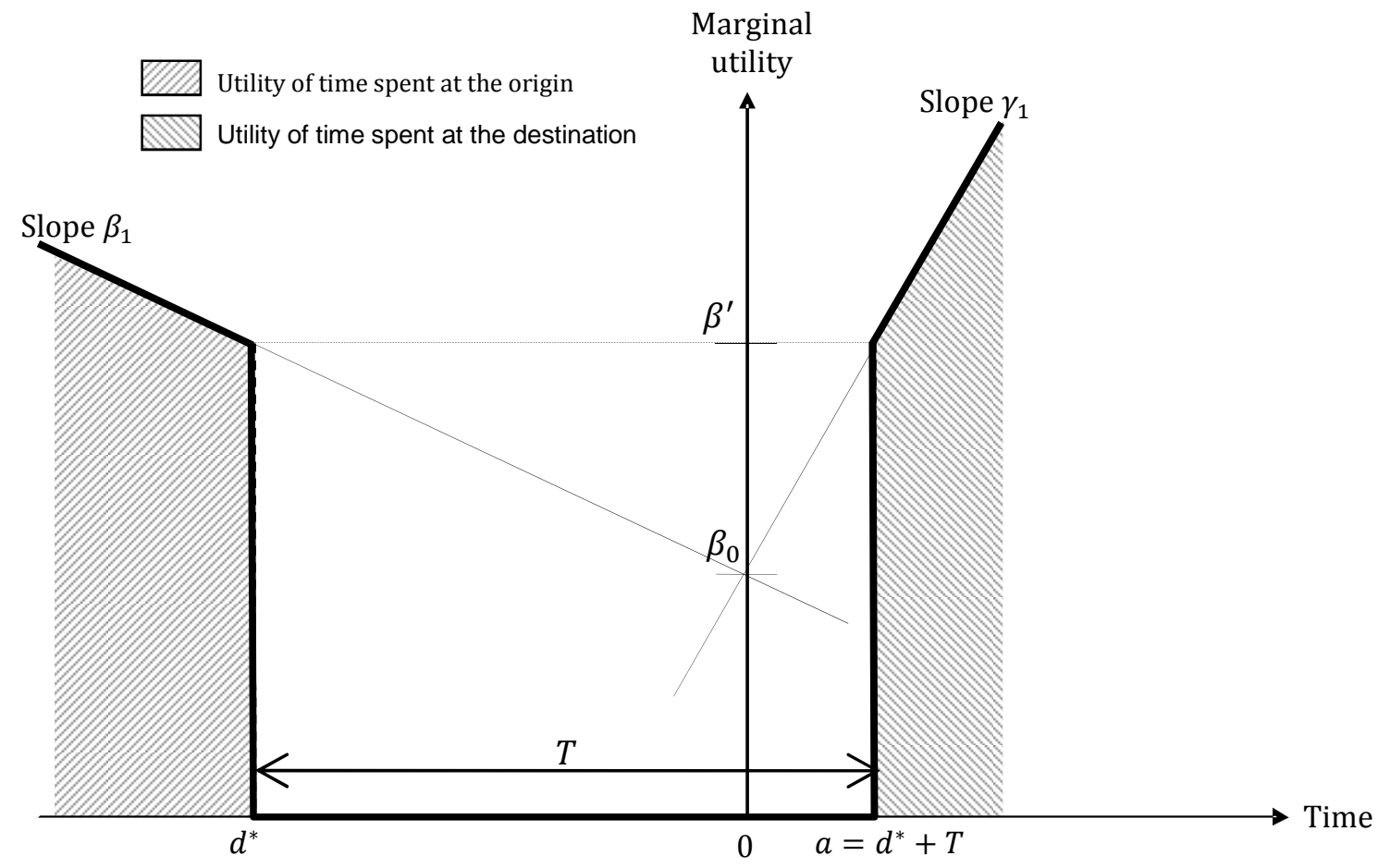

Figure 2. Marginal utilities of the Slope Model. Shaded areas show total utility. The figure is drawn assuming optimal departure time.

As opposed to the Step Model, there is no natural "preferred arrival time", when the marginal utility at the destination suddenly rises. Instead, the optimal arrival time (and consequently the optimal departure time) is determined by the condition that the marginal utility at the origin at the time of departure must be equal to the marginal utility at the destination at the time of arrival, such that utility is maximized. Given a fixed travel time $T$, the optimal departure time $d^{*}$ can be found by maximizing $u(d, d+T)$ from (11) (Fosgerau \& Engelson, 2011), which gives:

$d^{*}=\frac{\gamma_{1}}{\beta_{1}-\gamma_{1}} T$

The corresponding maximal utility $u^{*}$ becomes:

$u^{*}=-\beta_{0} T-\frac{\gamma_{1}}{2}\left(d^{*}+T\right)^{2}+\frac{\beta_{1}}{2}\left(d^{*}\right)^{2}$

In the first stated choice experiment, each choice alternative consists of some deviation $\Delta d$ in the departure time and some deviation $\Delta a$ in the arrival time relative to the reference trip. Assuming that the reference trip was chosen optimally by the respondent, these deviations translate into a loss of utility $\Delta u$ relative to the reference trip. Using equations (11)-(13), the difference in utility between a stated choice alternative $(\Delta d, \Delta a)$ and the reference trip becomes (see Appendix B for details):

$\Delta u=-\left(\beta_{0}+\beta_{1} \frac{\gamma_{1}}{\beta_{1}-\gamma_{1}} \cdot T\right) \cdot(\Delta a-\Delta d)-\frac{\gamma_{1}}{2} \cdot(\Delta a)^{2}+\frac{\beta_{1}}{2} \cdot(\Delta d)^{2}$. 
Each stated choice question is a choice between two such $\Delta u$ 's. The travel time $T$ refers specifically to the travel time of the reference trip, and hence does not vary across choices in the experiment.

\section{Reduced Form}

Now, assume that the travel time $T$ is a random variable, with $E(T)=\mu$ and $\operatorname{Var}(T)=\sigma^{2}$. Fosgerau and Engelson $(2011)^{5}$ show that the traveller's optimal departure time $d^{*}$ is given by:

$d^{*}=\frac{\gamma_{1}}{\beta_{1}-\gamma_{1}} \mu$,

Note that this quantity is independent of $\sigma$, the optimal departure time given in (15) is the same as in the deterministic case (12), except that the deterministic travel time $T$ is replaced by the mean $\mu$. The fact that the optimal departure time is independent of the variability of the travel time rests upon the assumption of linearly changing marginal utilities at the origin and destination. Fosgerau and Engelson also show that the maximal expected total utility becomes

$u^{*}=-\beta_{0} \mu-\frac{\beta_{1} \gamma_{1}}{2\left(\beta_{1}-\gamma_{1}\right)} \mu^{2}-\frac{\gamma_{1}}{2} \sigma^{2}$

Note that $u^{*}$ does not depend on the full distribution of $T$, only on its mean and variance, in contrast to what we found in the Step Model case.

In the first choice experiment, we estimate the parameters $\beta_{0}, \beta_{1}$ and $\gamma_{1}$ from (14). In the second choice experiment, we estimate the a set of parameters $\psi$ in the following reduced-form expression:

$u=-\psi_{1} \mu-\psi_{2} \mu^{2}-\psi_{3} \sigma^{2}$

If the slope model correctly describes the respondents' choices, the marginal utilities of mean travel time $\mu$ and variance $\sigma^{2}$ should coincide between the two choice experiments. These marginal utilities are obtained by differentiating (16) and (17) with respect to $\mu$ and $\sigma^{2}$, respectively. Hence, we should expect the following two conditions to hold:

$\beta_{0}+\frac{\beta_{1} \gamma_{1}}{\left(\beta_{1}-\gamma_{1}\right)} \mu=\psi_{1}+2 \psi_{2} \mu$, and $\frac{\gamma_{1}}{2}=\psi_{3}$.

\subsection{Summary of Scheduling Models}

Table 1 summarizes the Step Model and the Slope Model - their scheduling specifications, the implied reduced forms, and the hypothesized equivalences between the first and second choice experiments.

\footnotetext{
${ }^{5}$ Our presentation differs slightly from Fosgerau and Engelson (2010) in that we do not, as they do, normalize the scale of utility such that $\beta_{1}=\gamma_{1}-1$; regardless, the presentations are equivalent.
} 
Table 1. Matrix of scheduling models, their reduced forms, and hypothesized equivalences between choice experiments.

\begin{tabular}{|c|c|c|c|}
\hline & & Model Specification & \\
\hline & & Step Model & Slope Model \\
\hline \multirow{2}{*}{$\begin{array}{l}\text { Scheduling } \\
\text { Preferences }\end{array}$} & $\begin{array}{l}\text { Marginal Utility } \\
\text { at Origin }\end{array}$ & Constant at $\alpha$ & $\begin{array}{l}\text { Linear with slope } \beta_{1} \text {, } \\
\text { intercept } \beta_{0}\end{array}$ \\
\hline & $\begin{array}{l}\text { Marginal Utility } \\
\text { at Destination }\end{array}$ & $\begin{array}{l}\text { Step function, jumping from } \\
\alpha-\beta \text { to } \alpha+\gamma\end{array}$ & $\begin{array}{l}\text { Linear with slope } \\
\gamma_{1}>\beta_{1} \text {, intercept } \beta_{0}\end{array}$ \\
\hline \multirow{2}{*}{$\begin{array}{l}\text { Reduced } \\
\text { Form }\end{array}$} & $\begin{array}{lr}\text { Utility } & \text { Term for } \\
\text { Mean } & \text { Travel } \\
\text { Time } & \end{array}$ & $-\alpha \mu$ & $-\beta_{0} \mu-\frac{\beta_{1} \gamma_{1}}{2\left(\beta_{1}-\gamma_{1}\right)} \mu^{2}$ \\
\hline & $\begin{array}{l}\text { Utility Term for } \\
\text { Variability }\end{array}$ & $-(\beta+\gamma) \sigma \int_{1-\frac{\beta}{\beta+\gamma}}^{1} \Phi^{-1}(s) d s$ & $-\frac{\gamma_{1}}{2} \sigma^{2}$ \\
\hline \multirow{2}{*}{$\begin{array}{l}\text { Hypothesized } \\
\text { equivalences } \\
\text { between choice } \\
\text { experiments }\end{array}$} & $\begin{array}{l}\text { Equal value of } \\
\text { travel } \\
\text { savings }\end{array}$ & $\theta_{1}=\alpha$ & $\begin{array}{l}\psi_{1}+\psi_{2} \mu \\
=\beta_{0}+\frac{\beta_{1} \gamma_{1}}{2\left(\beta_{1}-\gamma_{1}\right)} \mu\end{array}$ \\
\hline & $\begin{array}{l}\text { Equal value of } \\
\text { travel time } \\
\text { variability }\end{array}$ & $\begin{array}{r}\theta_{2}^{p_{1}}=\alpha+\gamma \\
\text { (for expected delay) }\end{array}$ & $\begin{array}{l}\psi_{3}=\frac{\gamma_{1}}{2} \\
\text { (for variance) }\end{array}$ \\
\hline
\end{tabular}

Note: $\mu$ is mean travel time, $\sigma$ is standard deviation of travel time, and $\Phi$ is the cumulative distribution of normalized travel times.

In the first pair of rows, the table expresses the underlying scheduling preference functions that form the basis of the scheduling form utilities. In the second pair of rows, the corresponding reduced form utilities are expressed in terms of the parameters from the scheduling functions. The last pair of rows sets out the hypothesized equivalences between the two forms of expressing utilities under each underlying scheduling preference function, where the scheduling parameters are obtained from an experiment related to specified departure and arrival times, and the $\theta$ - and $\psi$ parameters are obtained from an experiment related to uncertain travel times. When it comes to testing these hypothesized equivalences using our two stated choice experiments, note that the parameter scales on either side of the equality are likely to differ, so the parameters cannot be compared directly across models. Rather, we will compare ratios between parameters, for example by dividing with the cost parameters of the respective models.

\section{THE DATA}

\subsection{Survey method}

The data originate from a stated choice study administered to travellers on the metro and commuter trains going toward and away from the centre of Stockholm city during the morning and afternoon peak periods. The respondents were recruited at selected stations on Monday-Thursday from 7-9 am and 4-6 pm during one week of October 2009. The respondents received questionnaires along with a pen and a stamped envelope. They were asked to fill in the questionnaire during the journey or shortly 
afterwards and to mail back the survey. In total, 3200 questionnaires were distributed, and the final data set comprised 1260 respondents, giving a response rate of $39 \%$.

The questionnaire began with questions about the observed trip (travel time, start time, constraints at origin/destination, transfers, safety margins (defined as the difference between the time constraint at the destination (if any) and the scheduled arrival time), frequency of delays etc.), partly to remind respondents about the circumstances of this particular trip.

The second part of the questionnaire comprised two different sets of binary stated choice experiments related to their actual journey. The first experiment was designed to estimate a scheduling model with non-random time deviations from the actual journey. The alternatives differed in three dimensions: fare, start time and travel time. As a consequence of the last two variables the alternatives also differed with respect to arrival time.

The second choice experiment was designed for estimating a reduced-form model, where the binary choices differed in delay length, delay risk, scheduled travel time (without delays) and fare. Hence, we present a binary travel time distribution to the respondents, where the travel time is $t$ with probability $1-p$ and $t+L$ with probability $p$.

In both experiments, the option to cancel the trip was also offered. In the estimation, the "cancel the trip" observations were first included, applying nested models with the choice of whether to accept any of the alternatives at the upper level and the choice between the right-hand and left-hand side at the lower level. This did not change parameter estimates for the scheduling model, while it almost doubled the value of delay time in the reduced-form models. This seemed to be because some respondents abstained from the trip once there were high delay risks. Due to a number of internal consistency problems with these model specifications, "abstain" answers were eventually dropped from the estimations. Examples of the binary choices are shown in Figure 3 and 


\begin{tabular}{|c|c|c|}
\hline & Departure 1 & Departure 2 \\
\hline $\begin{array}{l}\text { Delay (if } \\
\text { you made } \\
\text { this trip } \\
\text { every day): }\end{array}$ & $\begin{array}{l}\text { Once every other month, the } \\
\text { train is } 45 \text { min delayed. } \\
\text { All other trips are on-time. }\end{array}$ & $\begin{array}{l}\text { Once every other week, the } \\
\text { train is } \mathbf{1 0} \text { min delayed. } \\
\text { All other trips are on-time. }\end{array}$ \\
\hline $\begin{array}{l}\text { Travel time } \\
\text { according to } \\
\text { the } \\
\text { timetable: }\end{array}$ & 3 min shorter than today & 10 min shorter than today \\
\hline Ticket price & $€ 0.20$ higher than today & $€ \mathbf{1 . 0 0}$ higher than today \\
\hline \multirow[t]{2}{*}{ I choose } & $\square 1$ & $\square 2$ \\
\hline & \multicolumn{2}{|c|}{ Cancel the trip $\square 3$} \\
\hline
\end{tabular}

Figure 4. Each questionnaire contained four binary choices from each of the two experiments. 


\begin{tabular}{|l|c|c|}
\hline \multirow{4}{*}{$\begin{array}{l}\text { Start Time } \\
\text { Travel Time }\end{array}$} & Departure 1 & Departure 2 \\
\cline { 2 - 3 } & $\mathbf{2 5}$ min later than today & $\mathbf{5}$ min later than today \\
\cline { 2 - 3 } Ticket Price & $\mathbf{1 5}$ min longer than today & $\mathbf{4 5}$ min longer than today \\
\cline { 2 - 3 } Arrival Time & (40 min later than today) & (50 min later than today) \\
\cline { 2 - 3 } I choose & \multicolumn{2}{|c|}{$\square 1$} \\
\cline { 2 - 3 } & \multicolumn{2}{|c|}{ Cancel the trip $\square 3$} \\
\hline
\end{tabular}

Figure 3: Survey question, choice experiment 1

\begin{tabular}{|c|c|c|}
\hline & Departure 1 & Departure 2 \\
\hline $\begin{array}{l}\text { Delay (if } \\
\text { you made } \\
\text { this trip } \\
\text { every day): }\end{array}$ & $\begin{array}{l}\text { Once every other month, the } \\
\text { train is } \mathbf{4 5} \text { min delayed. } \\
\text { All other trips are on-time. }\end{array}$ & $\begin{array}{l}\text { Once every other week, the } \\
\text { train is } \mathbf{1 0} \text { min delayed. } \\
\text { All other trips are on-time. }\end{array}$ \\
\hline $\begin{array}{l}\text { Travel time } \\
\text { according to } \\
\text { the } \\
\text { timetable: }\end{array}$ & 3 min shorter than today & 10 min shorter than today \\
\hline Ticket price & $€ 0.20$ higher than today & $€ 1.00$ higher than today \\
\hline I choose & $\square 1$ & $\square 2$ \\
\hline & \multicolumn{2}{|c|}{ Cancel the trip $\square 3$} \\
\hline
\end{tabular}

Figure 4: Survey question, choice experiment 2

\subsection{Experimental Design}

An orthogonal pivot design was used for the two choice experiments. For both experiments, the difference between each of the factors in the binary choice alternatives was constructed using an orthogonal design table with 16 rows and one column for each factor. As explained above, the first experiment included three factors and the second four factors. In both experiments, all factor differences took four levels. The absolute level of each factor facing the respondents took many more levels, but this is irrelevant in the estimation. Simulation over a wide range of model specifications and parameter values, which also included the parameter values that were achieved in the pilot and the main study, was undertaken. This guarantees sufficient efficiency in parameter estimates and that the design can retrieves properties of the data assuming a variety of underlying model specifications. 
Eight different questionnaires with eight choice situations (four for each of two experiments) were constructed. Each of these was also mirrored, such that the left and right hand alternatives were switched, to investigate whether there is a greater tendency to choose the left hand alternative; no such tendency was found in the analysis. The choice alternatives are summarized in Table 2.

Table 2: Summary of the variables in the stated choice experiments. Departure, travel, arrival and travel time is given in [min] and fare in $[€]^{6}$. All variables are pivot values, apart from delay length and risk.

\begin{tabular}{|l|r|r|c|}
\hline & \multicolumn{1}{l|}{ Min. } & \multicolumn{1}{l|}{ Mean } & Max. \\
\hline Departure time, exp. 1 & -45 & -0.2 & 47 \\
\hline Travel time, exp. 1 & -6 & 9 & 45 \\
\hline Fare, exp. 1 [€] & -1 & -0.1 & 1.5 \\
\hline Arrival time, exp. 1 & -49 & 9 & 50 \\
\hline Travel time, exp. 2 & -10 & 3.5 & 22 \\
\hline Fare, exp. 2 [€] & -0.5 & 0.4 & 1.2 \\
\hline Delay length, exp. 2 & 5 & 25 & 70 \\
\hline Delay risk, exp. 2 & 0.025 & 0.08 & 0.2 \\
\hline
\end{tabular}

\subsection{Sample Statistics}

Table 3 and Table 4 summarize the descriptive statistics of the sample. The purpose at the destination was 'work' for 61 percent of the respondents and 'home' for 17 percent. The remaining 22 percent had some other purpose at the destination. 49 percent state that they had a time constraint at the destination. The mean door-to-door travel time was 46 minutes.

For those respondents who had a specific time constraint at the destination, their scheduled arrival times were, on average, 15 minutes prior to that time constraint.

Table 5 shows experiences of frequency of delays stated by respondents who make regular trips. The tabulated frequencies correspond to the levels of risk of delay included in the stated choice experiment, which according to the table are realistic. Table 5 also shows that about half of the respondents do not have a connection from the subway/commuter train to reach their final destination. The vast majority of the respondents having a connection have a connection with high frequency.

\footnotetext{
${ }^{6}$ In this table and throughout the paper we have converted SEK to Euro using a conversion rate of 10 SEK/€.
} 
Table 3: Sample statistics for categorical variables.

\begin{tabular}{|l|r|}
\hline \multicolumn{2}{|l|}{ Shares of the total sample population } \\
\hline Travellers recruited on the subway & 0.45 \\
\hline Travellers with constraint at the origin & 0.20 \\
\hline Travellers with constraint at the destination & 0.49 \\
\hline Women & 0.65 \\
\hline Children <13 years in the household & 0.29 \\
\hline Employed & 0.81 \\
\hline
\end{tabular}

\begin{tabular}{|l|r|}
\hline \multicolumn{2}{|l|}{ Shares of purposes at destination } \\
\hline Work & 0.61 \\
\hline School & 0.06 \\
\hline Business & 0.02 \\
\hline Shopping & 0.03 \\
\hline Recreation & 0.03 \\
\hline Other & 0.08 \\
\hline Home trip & 0.17 \\
\hline
\end{tabular}

Table 4: Sample statistics for continuous variables.

\begin{tabular}{|l|l|l|l|l|l|l|}
\hline & Min. & $\begin{array}{l}\text { 1st } \\
\text { quartile }\end{array}$ & Mean & $\begin{array}{l}\text { 3rd } \\
\text { quartil } \\
\text { e }\end{array}$ & Max. & n/a \\
\hline Door-to-door travel time [min] & 0 & 30 & 46 & 55 & 580 & 39 \\
\hline $\begin{array}{l}\text { Safety margin, travellers with } \\
\text { constraint at destination [min] }\end{array}$ & 0 & 10 & 15 & 20 & 100 & 703 \\
\hline Age [years] & 18 & 34 & 44 & 55 & 87 & 69 \\
\hline Monthly income before tax [€] & 500 & 2250 & 2850 & 3750 & 7000 & 77 \\
\hline
\end{tabular}

Table 5: Stated frequency of delays and headway of connection to final destination, as shares of the total sample.

\begin{tabular}{|l|r|l|}
\hline \multicolumn{3}{|l|}{ Stated frequency of delays for regular trips } \\
\hline & Subway & \multicolumn{2}{l|}{$\begin{array}{l}\text { Commuter } \\
\text { train }\end{array}$} \\
\hline Once per week & 0.14 & 0.19 \\
\hline Every other week & 0.13 & 0.15 \\
\hline Once per month & 0.18 & 0.26 \\
\hline $\begin{array}{l}\text { Every other month / } \\
\text { less }\end{array}$ & 0.30 & 0.25 \\
\hline Not a regular trip & 0.26 & 0.15 \\
\hline
\end{tabular}

\begin{tabular}{|l|l|}
\hline \multicolumn{2}{|l|}{ Headway of connection } \\
\hline$<5 \mathrm{~min}$ & 0.17 \\
\hline $10 \mathrm{~min}$ & 0.15 \\
\hline $15 \mathrm{~min}$ & 0.13 \\
\hline $20 \mathrm{~min}$ & 0.04 \\
\hline 1 hour or more & 0.02 \\
\hline Don't know & 0.03 \\
\hline $\begin{array}{l}\text { Don't have } \\
\text { connection }\end{array}$ & 0.45 \\
\hline
\end{tabular}

\section{ESTIMATION}

In this section, we present estimation results for the two scheduling models and the two corresponding reduced-form models. As explained in Section 2, we denote the two 
scheduling models the "step model" and the "slope model". In all of the estimations, we estimate binary logit models. The left-hand alternative is chosen if:

$u_{L}-u_{R}+\varepsilon \geq 0$

where $\varepsilon$ is taken to be an i.i.d. logistic error term and $u_{L}$ and $u_{r}$ are the measurable utilities of the left- and right-hand alternatives given in each survey question.

\subsection{Experiment 1: Scheduling models}

The step model specification was given in (2). Adding the travel cost $c$ with a marginal utility of $\lambda$, we estimate the utility function of the left and right hand alternative as follows:

$u(d, a)=-\alpha(a-d)+\beta \min (0, a)-\gamma \max (0, a)-\lambda c$,

where time 0 is taken to be the respondent's actual arrival time. Respondents were also asked whether they had to be in time for anything specific at the destination, and if so, how much margin they had before that time. However, replacing the actual arrival time with this time yielded considerably worse estimation results.

The slope model is defined by (13). The utility function of the left and right hand alternatives represent the deviation from the reference trip. We assume that the reference trip (the trip on which the respondent was recruited) was the respondent's optimal choice. Letting $\Delta d$ and $\Delta a$ be the changes in departure time and arrival time relative to the reference trip, the change in utility $\Delta u$ becomes:

$\Delta u=-\left(\beta_{0}+\beta_{2} T\right) \cdot(\Delta a-\Delta d)-\frac{\gamma_{1}}{2} \cdot(\Delta a)^{2}+\frac{\beta_{1}}{2} \cdot(\Delta d)^{2}-\lambda c$,

where $\beta_{2}=\frac{\beta_{1} \gamma_{1}}{\beta_{1}-\gamma_{1}}$ should hold theoretically according to (14). ${ }^{7} \beta_{0}$ is a random parameter, different across individuals, that captures panel effects. Estimation results for the two scheduling models are found in Table 6.

${ }^{7}$ Imposing the constraint $\beta_{2}=\frac{\beta_{1} \gamma_{1}}{\beta_{1}-\gamma_{1}}$ in the estimation causes $\beta_{1}$ and $\gamma_{1}$ to pick up intra-individual preference variation, since $T$ is the travel time of the reference trip and hence only varies across individuals, not across choices. A biased estimate of $\gamma_{1}$ is a serious problem, since $\gamma_{1}$ translates to the value of variance which will be compared to the corresponding value from the reduced-form model the main purpose of this paper. Letting $\beta_{0}$ be a random parameter, constant within individuals, partially solves this problem, but the precision of the $\gamma_{1}$ estimate remains considerably worse than in a model where $\beta_{2}$ is estimated as a free parameter. For this reason, we have chosen to estimate $\beta_{2}$ as a free parameter and $\beta_{0}$ as a normally distributed random parameter constant within individuals (which has the added benefit of picking up panel effects). Imposing the constraint $\beta_{2}=\frac{\beta_{1} \gamma_{1}}{\beta_{1}-\gamma_{1}}$ does not change the conclusions of the paper; estimation results for this model are available on request from the authors. 
Table 6: Estimation Results of the Scheduling Models. The values of time at destination and origin implied by the slope mode are computed as the average of the first 15 minutes of deviation from the reference situation. All values of time are translated into $€ /$ hour.

\begin{tabular}{|c|c|c|c|c|}
\hline \multirow[b]{2}{*}{ Parameter (unit) } & \multicolumn{2}{|c|}{ Step Model } & \multicolumn{2}{|c|}{ Slope Model } \\
\hline & Value & t-stat & Value & $t$-stat \\
\hline$\lambda(/ \mathrm{SEK})$ & 0.8222 & 14.32 & 1.21 & 15.98 \\
\hline$\alpha(/ \min )$ & 0.0919 & 17.19 & & \\
\hline$\beta(/ \min )$ & 0.0622 & 16.73 & & \\
\hline$\gamma(/ \mathrm{min})$ & 0.0579 & 13.43 & & \\
\hline Mean $\beta_{0}(/ \min )$ & & & 0.16 & 10.44 \\
\hline $\operatorname{Std} \operatorname{dev} \beta_{0}(/ \mathrm{min})$ & & & 0.0723 & 9.85 \\
\hline$\beta_{2}(/ \min )$ & & & -0.00016 & -0.57 \\
\hline$\beta_{1}\left(/ \mathrm{min}^{2}\right)$ & & & -0.00273 & -10.61 \\
\hline$\gamma_{1}\left(/ \min ^{2}\right)$ & & & 0.000837 & 2.8 \\
\hline Model Statistics & \multicolumn{2}{|c|}{ Step Model } & \multicolumn{2}{|c|}{ Slope Model } \\
\hline Observations & & 3226 & \multicolumn{2}{|r|}{3226} \\
\hline Final $\log (L)$ & & -1666.7 & \multicolumn{2}{|r|}{-1585.96} \\
\hline No. Parameters & & 4 & \multicolumn{2}{|r|}{5} \\
\hline$\rho^{2}(0)$ & & 0.255 & \multicolumn{2}{|r|}{0.291} \\
\hline No. Draws & & & \multicolumn{2}{|r|}{1000} \\
\hline $\begin{array}{l}\text { Value of early departure } \\
\text { time }\end{array}$ & \multicolumn{2}{|l|}{$\frac{\alpha}{\lambda}=6.7$} & $\frac{1}{\lambda}\left(\beta_{0}-\frac{15 \beta_{1}}{2}\right)$ & $=6.9$ \\
\hline Value of late departure time & \multicolumn{2}{|l|}{$\frac{\alpha}{\lambda}=6.7$} & $\frac{1}{\lambda}\left(\beta_{0}+\frac{15 \beta_{1}}{2}\right)$ & $=8.9$ \\
\hline $\begin{array}{l}\text { Value of early arrival time, } \\
\text { average of the first } 15 \mathrm{~min}\end{array}$ & \multicolumn{2}{|c|}{$\frac{\alpha-\beta}{\lambda}=2.2$} & $\frac{1}{\lambda}\left(\beta_{0}-\frac{15 \gamma_{1}}{2}\right)$ & $=7.6$ \\
\hline $\begin{array}{l}\text { Value of late arrival time, } \\
\text { average of the first } 15 \mathrm{~min}\end{array}$ & \multicolumn{2}{|c|}{$\frac{\alpha+\gamma}{\lambda}=10.9$} & $\frac{1}{\lambda}\left(\beta_{0}+\frac{15 \gamma_{1}}{2}\right)$ & $=8.2$ \\
\hline
\end{tabular}

All parameters have the expected sign and relative magnitude $(\beta<\alpha$ in the step model and $\beta_{1}<\gamma_{1}$ in the slope model). The slope model fits the data better than the step model: a multinomial logit slope model (i.e. not accounting for panel effects - this 
model is not presented in the table) has a better likelihood than the step model (1620.2 compared to -1666.7), with the same number of parameters. The better model fit of the slope model stems from relaxing the step model's restriction of constant marginal utility of time at the origin (relative to the marginal utility of travelling) ${ }^{8}$. This is also evident from the finding that $\gamma_{1}<-\beta_{1}$ in the slope model, implying that the marginal utility of time at the origin changes faster than the marginal utility of time at the destination. This indicates that some respondents have constraints at the origin that are less flexible than the constraints at the destination. In the step model, this is reflected by the finding that the $\gamma$ parameter is fairly small relative to the $\alpha$ parameter, meaning that the additional utility of being at the destination after the PAT, rather than being at the origin, is not very large.

The valuation of marginal changes in the arrival and departure times are compared at the bottom of Table 5. These reflect the willingness to pay for moving the departure/arrival time backwards or forwards, keeping arrival/departure at the other trip end constant ${ }^{9}$. In the slope model, the valuations of marginal changes in the arrival/departure times are not constant: we have chosen to evaluate as the average valuation 15 minutes before and after the reference point, to make them reasonably comparable with the corresponding valuations in the Step Model. The difference between the valuations of an earlier and a later arrival is much larger in the Step Model than in the Slope Model; in other words, the slope of the marginal utility at the destination in the Slope Model does not nearly correspond to the jump in marginal utility of the Step Model. The difference is so large partly because of the Step Model's restriction of constant marginal utility of time at the origin. This affects the other estimates because departure and arrival times are correlated, exaggerating the difference in utility between early and late arrival time (relaxing the restriction of constant marginal utility of time at the origin reduces the size of the jump in the marginal utility at the destination).

The two scheduling models are illustrated and compared with an example in Figure 5. The figure depicts how the marginal utilities of the two models change with clock time as the traveller spends time at the origin, travels, and subsequently spends time at the destination. The example shows a traveller departing 30 minutes before PAT and arriving 10 minutes before PAT. The marginal utilities are expressed in monetary terms by dividing them with the respective cost parameter, to make them comparable between models ${ }^{10}$.

\footnotetext{
${ }^{8}$ If estimating the step model without the restriction of constant marginal utility of time at the origin, the log likelihood value improves to -1596 which indicates a better model fit than the slope model estimated as an ordinary logit mode with log likelihood value -1620.2.

${ }^{9}$ These values are derived from the utility functions (19.) and (20.). The value of a change of departure time $d$ is $\frac{\partial u}{\partial d} / \frac{\partial u}{\partial c}$ in the Step Model (using (19.)), and $\frac{\partial \Delta u}{\partial \Delta d} / \frac{\partial \Delta u}{\partial c}$ in the Slope Model (using (20.)). The value of a change of arrival time is $\frac{\partial u}{\partial a} / \frac{\partial u}{\partial c}$ in the Step Model, and $\frac{\partial \Delta u}{\partial \Delta a} / \frac{\partial \Delta u}{\partial c}$ in the Slope Model.

${ }^{10}$ In other words, they are computed as $\frac{\partial u}{\partial a} / \frac{\partial u}{\partial c}$ (Step Model) and $\frac{\partial \Delta u}{\partial \Delta a}$ (Slope Model) at the destination, and $\frac{\partial u}{\partial d} / \frac{\partial u}{\partial c}$ (Step Model) and $\frac{\partial \Delta u}{\partial \Delta d} / \frac{\partial u}{\partial c}$ (Slope Model) at the origin.
} 


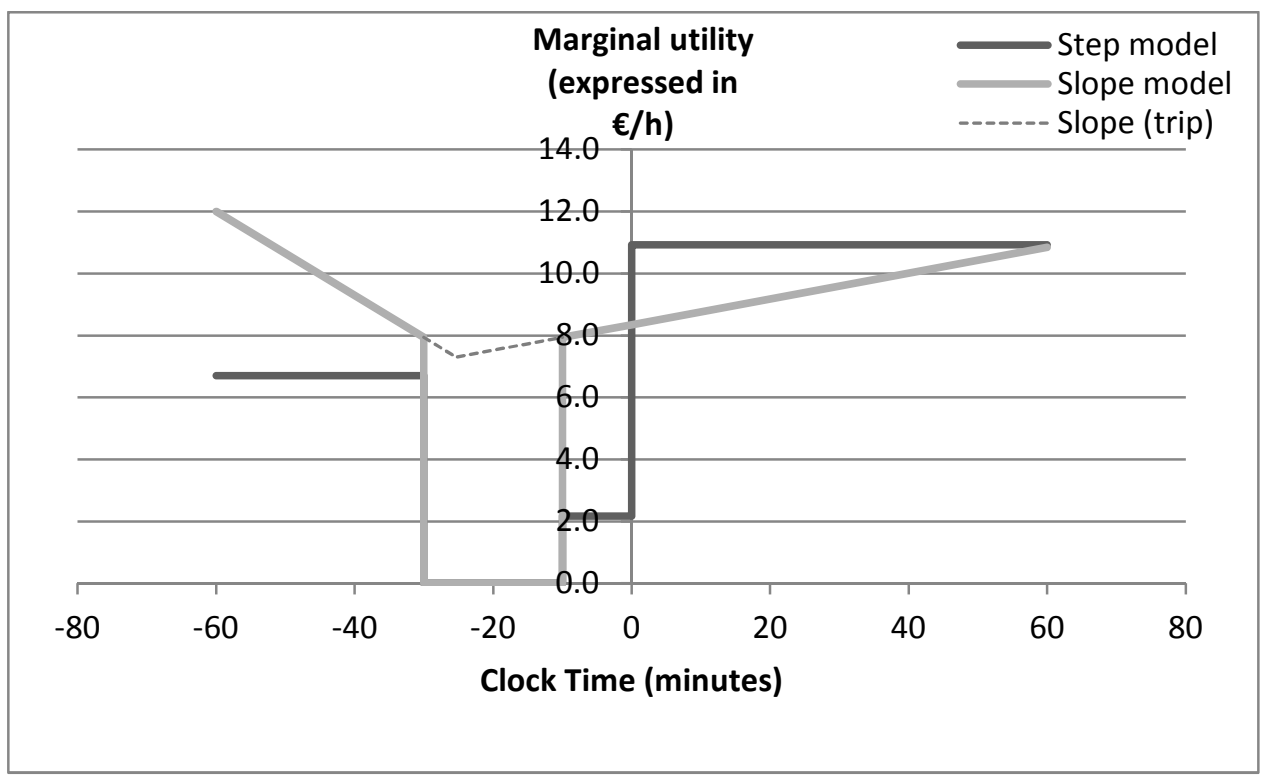

Figure 5. Illustration of how the marginal utility of time changes with clock time in the Step and Slope scheduling models, assuming an example trip.

Some travellers stated that they had constraints at the origin or destination. Interestingly, we could not find any differences in scheduling parameters between these respondents and respondents stating that they had no specific scheduling constraints. A possible interpretation is that most individuals have constraints to some extent, but these may seldom be absolutely "binding". This interpretation would explain the better performance of the slope model, which does not assume binding constraints at specific points in time, but rather a gradually increasing or decreasing marginal utility of time.

\subsection{Experiment 2: Reduced-form models}

As explained in Section 2, the two scheduling models imply different reduced-form models, which are both estimated using data from the second choice experiment. Remember that we use a binary travel time distribution where the travel time is $t$ with probability $(1-p)$, whereas a delay of length $L$ occurs with probability $p$. This means that the travel time distribution's mean $\mu$ and variance $\sigma^{2}$ are:

$\mu=t+p L ; \quad \sigma^{2}=p(1-p) L^{2}$.

To estimate the reduced-form Step Model we apply

(7), estimating a separate parameter for each risk level $p$ in the stated choice experiment, $\theta_{2}^{p=0.025}, \theta_{2}^{p=0.05}, \theta_{2}^{p=0.10}$ and $\theta_{2}^{p=0.20}$, such that the utility function of each alternative becomes:

$u=-\theta_{1} t-\left(\theta_{2}^{p=0.025}+\theta_{2}^{p=0.05}+\theta_{2}^{p=0.10}+\theta_{2}^{p=0.20}\right) p L-\lambda c$,

The reduced-form expression corresponding to the Slope Model is specified in (17), so the utility function becomes:

$u=-\psi_{1}(t+p L)-\psi_{2}(t+p L)^{2}-\psi_{3} p(1-p) L^{2}-\lambda c$, 
Estimation results are found in All parameters have the expected sign and relative magnitude. The $\theta_{2}$ parameters in the Step Model increase with the risk level, as expected, except for $\theta_{2}^{p=0.05}$ and $\theta_{2}^{p=0.10}$, but these are not significantly different. The Step Model has two more parameters and somewhat better goodness-of-fit. The additional two parameters do, however, not significantly improve the model according to the $\chi^{2}$ test of parameter restriction.

Table 7. All parameters have the expected sign and relative magnitude. The $\theta_{2}$ parameters in the Step Model increase with the risk level, as expected, except for $\theta_{2}^{p=0.05}$ and $\theta_{2}^{p=0.10}$, but these are not significantly different. The Step Model has two more parameters and somewhat better goodness-of-fit. The additional two parameters do, however, not significantly improve the model according to the $\chi^{2}$ test of parameter restriction. 
Table 7: Estimation Results of the two Reduced-Form Models.

\begin{tabular}{|c|c|c|c|c|}
\hline & \multicolumn{2}{|c|}{ Step Model } & \multicolumn{2}{|c|}{ Slope Model } \\
\hline & Value & t-stat & Value & $t$-stat \\
\hline$\lambda$ & 1.07 & 6.9 & 1.54 & 13.7 \\
\hline$\theta_{1}$ and $\psi_{1}$ & 0.107 & 5.4 & 0.095 & 4.6 \\
\hline$\theta_{2}^{p=0.025}$ & 0.805 & 5.6 & & \\
\hline$\theta_{2}^{p=0.05}$ & 0.634 & 6.8 & & \\
\hline$\theta_{2}^{p=0.10}$ & 0.686 & 7.6 & & \\
\hline$\theta_{2}^{p=0.20}$ & 0.565 & 8.8 & & \\
\hline$\psi_{2}$ & & & 0.0010 & 3.1 \\
\hline$\psi_{3}$ & & & 0.0050 & 5.4 \\
\hline Observations & 2996 & & 2996 & \\
\hline Final $\log (L)$ & -1781.58 & & -1783.4 & \\
\hline No. Parameters & 6 & & 4 & \\
\hline$\rho^{2}(0)$ & 0.1421 & & 0.1412 & \\
\hline $\begin{array}{l}\text { Value of travel time savings } \\
(€ / h)\end{array}$ & 6.0 & & 7.4 & $\begin{array}{r}\text { Evaluated at } \\
p=0.08, L=25 \\
\text { (design means) }\end{array}$ \\
\hline $\begin{array}{l}\text { Value of expected delay } \\
(€ / h) \text { : }\end{array}$ & & & & $\begin{array}{r}\text { Evaluated at } L=25, \\
t=46 \text { (design and } \\
\text { sample means) }\end{array}$ \\
\hline$p=0.025$ & 45.1 & & 22.8 & \\
\hline$p=0.05$ & 35.6 & & 23.0 & \\
\hline$p=0.10$ & 38.5 & & 23.4 & \\
\hline$p=0.20$ & 31.7 & & 24.2 & \\
\hline
\end{tabular}

The Step Model assumes that the disutility of a delay is linear in $L$. Intuitively, this might be unexpected - but in fact, this is supported by the estimation results. Testing various piecewise linear functions, we find no evidence that the marginal value of delay length varies with the delay length itself. 
Table 7 also compares the value of scheduled travel time $t$ and the value of expected delay $p L$ between the two reduced-form models. Using we have that the value of scheduled travel time is:

$$
-\frac{1}{\lambda} \frac{\partial u}{\partial t}=\frac{\theta_{1}}{\lambda}
$$

in the step model. From (22) we have that the corresponding value in the slope model is:

$$
-\frac{1}{\lambda} \frac{\partial u}{\partial t}=\frac{-\psi_{1}-\psi_{2} * 2(p L+t)}{\lambda}
$$

The latter depends on on $p, L$ and $t$; it is evaluated at sample and design means $t=46$, $p=0.08, L=25$. The values of scheduled travel time turn out to be similar in the two models.

Meanwhile, the value of expected delay in the step model is:

$$
-\frac{1}{\lambda} \frac{\partial u}{\partial(p L)}=\frac{\theta_{2}^{p}}{\lambda}
$$

in the step model, and in the slope model it is:

$$
-\frac{1}{\lambda} \frac{\partial u}{\partial(p L)}=\frac{-\psi_{1}-\psi_{2} * 2(t+p L)-\psi_{3}(1-2 p) L}{\lambda}
$$

The latter is also evaluated at sample and design means. The slope model produces lower values of expected delay than the step model. If however, all $\theta_{2}$ 's in the Step Model are constrained to be equal, the value of expected delay becomes similar to that produced by the slope model. In other words, when the model does not allow the value of delay time to depend on $p$, the value decreases considerably, and this explains the relatively low valuation in the Slope Model.

Just as in the scheduling models, the valuation of delays is not different for respondents which stated that they had arrival or departure time constraints.

\subsection{Other remarks on estimation results}

From the extensive testing of model specification that was carried out, the following observations can be noted:

- There are no significant differences in scheduling parameters between morning and afternoon trips, or between different trip purposes.

- There are only small differences in preferences between metro and commuter train travellers. The only significant difference is that commuter train travellers value time at the origin higher. A likely reason for this is that commuter trains are more crowded; the traveller's door-to-door travel times do not differ between the models. This difference is only significant in the first experiment. This was not included in the final model, however.

- People who stated that they had constraints on their arrival or departure time did not in fact have significantly larger scheduling parameters than the rest of the 
respondents. Nor did people with constraint on their arrival have a higher valuation of expected delay.

- The model using the observed arrival time as preferred arrival times gave a better model fit and response scale than alternative models where the arrival times were adjusted by respondents' stated "safety margin" (if any).

- Having a connecting trip leg at the end of the recruitment trip leg did not affect valuations.

- Higher income attenuates the cost parameter.

- Once income differences are controlled for, no gender differences are found.

- Surprisingly, we found that parents of both sexes with children 12 years or younger have a higher valuation of time but not a higher valuation of expected delay or larger scheduling parameters. A reason might be that there are very few trips with purpose "fetch the children from day care" in the sample.

- Employed persons have a higher valuation of lateness than the unemployed.

- There is no significant constant for the left-hand alternative in any of the experiments. Hence, no tendency was found that respondents "cheated" on a difficult task by always selecting the same side.

Mixed logit (MXL) models have been estimated, to ensure that the results are robust and to take panel effects into account. In the first MXL model of the first experiment, all parameters were assumed to be normally distributed. The valuations, computed using the mean values of the parameters, remained robust as compared to the MNL model. There was no significant variation between individuals in the $\alpha$ parameter. The largest random variation was found in the lateness parameter.

Assuming normally distributed parameters, fairly large parts of the mass of the distribution of the parameters were positive. To remedy this problem, the parameters were assumed symmetrically triangular and constrained to be negative. This model, on the other hand, turned out unsatisfactory, resulting in very high valuations. Presumably, there is a significant mass close to zero, which is better accounted for by using the normal distribution. The option of using log-normally distributed parameters was not tested, since the valuations are normally sensitive to assumptions about the long and flat tail where the data do not cover the support of the distribution.

Mixed logit models estimated for experiment 2 give the same results as for experiment 1. The heterogeneity is largest for the expected delay parameters. The valuations are robust assuming normally distributed parameters. The triangular distributions give very high valuations that probably indicate misspecification.

\section{DISCUSSION}

Based on the estimation results in the previous section, we are now able to explore the transferability between the scheduling approach and the reduced-form approach using the relationships derived in section 2.1 and 2.2 and assessing the hypothesized equivalences from Table 1, which is the key issue of this paper. The empirical comparisons are shown in Table 8. Since the scale of the models (the variance of the error term) is likely to be different across estimations, it is not meaningful to compare the absolute parameter values, but rather the ratios between corresponding parameters from the same model. For this reason we make the comparisons in 
monetary terms (dividing by the cost parameter), or relative to travel time (dividing with the marginal utility of travel time).

Section 2.3 summarized the conditions we expect to hold, if the results from the two choice experiments are consistent. For the Step Model, we should expect that $\theta_{1}=\alpha$ and $\theta_{2}^{p=0.025}=(\alpha+\gamma)$. For the Slope Model, we should expect that $\psi_{1}+2 \psi_{2} \mu=\beta_{0}+$ $2 \beta_{2} \mu$ and $\psi_{3}=\frac{\gamma_{1}}{2}$. In making the comparisons, we evaluate each expression using at the sample mean values $t=46, p=0.08, L=25$ such that $\mu=46+25 \cdot 0.08$. The resulting comparisons are presented in Table 8.

Table 8: Parameter Comparisons Between Scheduling and Reduced-Form Specifications. All values are given in $€ / h$.

\begin{tabular}{|c|c|c|c|c|}
\hline \multirow[b]{2}{*}{$\begin{array}{l}\text { Hypothesized } \\
\text { Equivalence }\end{array}$} & \multicolumn{2}{|c|}{ Step Model } & \multicolumn{2}{|c|}{ Slope Model } \\
\hline & $\begin{array}{c}\text { Scheduling } \\
\text { specification }\end{array}$ & $\begin{array}{l}\text { Reduced-form } \\
\text { specification }\end{array}$ & $\begin{array}{l}\text { Scheduling } \\
\text { specification }\end{array}$ & $\begin{array}{l}\text { Reduced-form } \\
\text { specification }\end{array}$ \\
\hline $\begin{array}{l}\text { Value of (expected) } \\
\text { travel time savings: }\end{array}$ & $\frac{\alpha}{\lambda}=6.7$ & $\frac{\theta_{1}}{\lambda}=6.0$ & $\frac{\beta_{0}+\beta_{2} \mu}{\lambda}=7.611$ & $\frac{\psi_{1}+2 \psi_{2} \mu}{\lambda}=7.4$ \\
\hline $\begin{array}{l}\text { Value of expected } \\
\text { delay: }(\alpha+\gamma) \sim \theta_{2}^{p=0.025}\end{array}$ & $\begin{array}{l}\frac{\alpha+\gamma}{\lambda}=10.9 \\
\frac{\alpha+\gamma}{\alpha}=1.6\end{array}$ & $\begin{array}{l}\frac{\theta_{2}^{p=0.025}}{\lambda}=45.1 \\
\frac{\theta_{2}^{p=0.025}}{\theta_{1}}=7.5\end{array}$ & & \\
\hline $\begin{array}{l}\text { Value of variance: } \\
\gamma_{1} / 2 \sim \psi_{3}\end{array}$ & & & $\begin{array}{c}\frac{\gamma_{1}}{2 \lambda}=0.021 \\
\frac{\gamma_{1}}{\beta_{0}+\beta_{2} \mu}=0.0026\end{array}$ & $\begin{array}{c}\frac{\psi_{3}}{\lambda}=0.19 \\
\frac{\psi_{3}}{\psi_{1}+2 \psi_{2} \mu}=0.026\end{array}$ \\
\hline
\end{tabular}

The first in each pairs of conditions suggests that the value of travel time savings should coincide in the scheduling model and the reduced-form model. As seen in Table 8 , the values of travel time savings are indeed very similar between the scheduling and reduced form models. This is true for both the Step and the Slope model. In this sense, all four models are reasonably consistent with each other.

The second condition in each pair of conditions suggests that the value of a certain measure of travel time variability should coincide in the scheduling model and the reduced-form model. For the Step Model, this measure turns out to be the expected delay $^{12}$. For the Slope Model, the variability measure that should be equivalent is the variance of the travel time. In contrast to the similarity of the value of travel time, the scheduling and the reduced-form models produce very different valuations of travel time variability - the reduced-form models consistently give considerably higher valuations.

\footnotetext{
${ }^{11}$ The parameter $\beta_{2}$ is set to zero, since it is insignificant and has the wrong sign in the estimation.

12 This is due to the specific travel time distribution we have used; in general, it is an expression involving the standard deviation of the travel time
} 
In the Step Model, the value of expected delay in the reduced-form model is more than 4 times larger than the corresponding value in the scheduling model. This is regardless of whether the comparison is made in monetary terms (dividing by the cost parameter) or relative to travel time (dividing by the travel time parameter). In the Slope Model, the reduced-form model produces a 9-10 times higher value of travel time variance than the scheduling model. Doing the comparison in monetary terms gives a slightly higher factor than comparing with the travel time.

Apparently, the scheduling models and the reduced-form models are inconsistent, in the sense that the estimated scheduling parameters in Experiment 1 cannot explain the value put on travel time variability in Experiment 2. In other words, the disutility of being subject to a risk of a delay exceeds what the scheduling models predict that the disutility should be. There seems to be some disutility associated with the uncertainty in Experiment 2, over and above the mere disutility of "late arrival" that is captured by the scheduling model in Experiment 1, where the arrival time is certain. In other words, being "delayed" seems to be worse than just being "late" in the sense of arriving after one's preferred arrival time.

Interestingly, the respondents do in fact answer the two experiments consistently in a specific sense: the respondents that have the highest disutility of late arrival in Experiment 1 are also the ones with the highest disutility of delay risk in Experiment 2. This was established using a bootstrap estimation, where the valuations of repeated sub-samples were estimated. This gave a correlation of 0.25 between the valuation of late arrival in Experiment 1 and the valuation of average delay in Experiment 2. On the other hand, no correlations were found for the cost or the (scheduled) travel time parameters. Apparently, the individual-specific variation in these parameters is swamped by random variation. This is consistent with the finding from the mixed-logit estimations, that the variations in the cost and time parameters are small compared to the variation in the value of expected delay.

So, it seems as if the discrepancy between the scheduling and reduced-form models cannot be explained by mere randomness. Instead, there may be several possible explanations of this phenomenon, and we will discuss some hypotheses that could explain why.

\subsection{Timing of information about the actual arrival time}

One of the differences between the two experiments is the point in time at which the traveller gets information about her actual arrival time. In experiment 1 , the traveller is given her arrival time in advance, and hence knows in advance whether she will be late with respect to the preferred arrival time (PAT). In experiment 2, only the probability and length of a delay is given, so the traveller does not know if she will actually be delayed when making a choice - in other words, information about the actual arrival time comes later than in experiment 1 . Under the theory of the scheduling model, this does not matter: the disutility of arriving after the PAT only depends on how late the traveller arrives - not how long in advance the actual travel time is known.

In reality, it is often the case that given ample warning, travellers can respond by adjusting their plans, thereby minimizing any lateness penalty they may incur by arriving late. In experiment 1 , the traveller is told that she will arrive after the PAT, and she can take measures accordingly, such as rescheduling activities in advance. In experiment 2 , if a delay actually occurs, activities have to be rescheduled with much 
shorter notice, if at all possible. Expressed in the terminology of the scheduling model, this means that the lateness penalty depends on how long in advance the traveller knows that he or she will be late. If information about the actual arrival time comes "early" (as in experiment 1), the lateness penalty will be lower than if the information comes "late" (as, potentially, in experiment 2). Put somewhat differently, "rescheduling costs" are bigger the later information comes.

Another way of putting it is that the notion of an exogenously fixed preferred arrival time may be untenable. For many travellers, the PAT may in fact be a wide interval, in which all arrival times are equally preferred - as long, that is, as the traveller knows in advance when she will arrive, and can plan accordingly. If this is the case, the "preferred" arrival time will be a function of when the traveller expects to arrive.

A natural question then is whether there is any way to present experiment 1 in such a way that the traveller does not get informed about the arrival time in advance (and thus can adjust her PAT). However, this is virtually impossible: one would have to ask respondents to rank alternatives that are essentially variants of "you would be delayed $\mathrm{X}$ minutes without knowing it in advance" - and at the same time, we want to assume that travellers choose the optimal departure time given an unknown travel time (knowing just its distribution). This is obviously not realistic. It is simply not possible to ask respondents whether they would prefer A or B - pretending at the same time that they would not know whether they had "chosen" A or B!

\subsection{The disutility of uncertainty per se}

In the scheduling model, there is no direct disutility of travel time uncertainty per se; disutility only arises from the risk of arriving late (and hence the need to start the trip earlier than otherwise). But it is possible that many people dislike not only the adjustments that might be made in the face of uncertainty, but also uncertainty in itself. This dislike can either be interpreted as an "anxiety cost", or as the "decision cost" needed to solve the optimal departure time problem. It is well established that information about the length of a delay is valued by travellers. This may be because it facilitates rescheduling of activities, but it may also be that the uncertainty per se is a concern for many travellers.

Another interpretation is that uncertainty creates a need for "contingency plans", if the traveller should be late; this special kind of planning cost is above and beyond the extra time needed to execute the contingency plan. Thus, the need to arrange a contingency plan, should one be delayed, is a cost created by the uncertainty per se, over and above the mere value of the (expected) delay.

In practice, anxiety costs, decisions costs and contingency planning costs are virtually inseparable from each other. On the other hand, separating them is not really necessary: what is important is that they all may cause a disutility exceeding the value of the mere costs of actual delays.

\subsection{Policy bias and focus bias}

In all stated preference experiments, there is a risk that valuations are overestimated due to policy bias or focus bias. "Policy bias" refers to respondents' tendency to answer in a way that they think will cause certain desirable effects - for example overstating their valuation of delays to make the transit authority try harder to decrease delays. "Focus bias" refers to respondents' tendency to forget other important characteristics 
of a trip, and hence unconsciously overstate their valuation of a certain aspect - in this case delays. Both these phenomena may be present in experiment 2 , since this dealt with delay risks explicitly.

\section{CONCLUSIONS}

The standard way to include the cost of travel time variability in appraisal is to estimate the parameters of a reduced-form expression including some measure of travel time variability - usually the standard deviation or the average delay. Different assumptions about the underlying scheduling model result in different forms of the reduced-form expression. The problem with such reduced-form approaches is that the valuation of variability will usually depend on the standardized travel time distribution. Since this distribution is likely to vary between contexts, a valuation estimated in one context cannot (consistently) be applied in another context.

One way to overcome this problem would be to estimate a scheduling model, and then derive the valuation in the reduced-form expression. This would also overcome the need to present travel time distributions to respondents in stated choice experiments, which is a difficult pedagogical task.

This approach has been tested in the present paper through comparing the results from two scheduling models and the corresponding reduced-form expressions. It turns out that the valuations of variability in the reduced-form models are much higher than the valuations implied by the scheduling models. The differences are so large that one may question the validity of the approach of motivating the standard reduced-form models from underlying scheduling models of the usual type. It seems unlikely that the valuations from the two models can be reconciled by mere tweaks of the models.

Instead, we are inclined to believe that the basic assumptions used when deriving a reduced-form model from an underlying scheduling model are not realistic - they do not capture enough of the essential features of the choice situation that travellers face. We hypothesize that there are two factors that are important to take into account.

First, the parameters of scheduling models, as they are usually interpreted and estimated, refer to a situation where the traveller gets information about his or her actual arrival time in advance (since respondents need to be aware of the attributes of the alternatives to make stated choice questions meaningful). This means that activities can be rescheduled to some extent, if necessary, to fit the actual arrival time. In contrast, when the travel time is random, the actual arrival time is not known until immediately before the arrival time. This makes rescheduling much more onerous, if possible at all, and hence the "lateness penalty" will be higher. Phrased differently, the parameters of the scheduling model, including the preferred arrival time, may depend on when traveller gets information about the actual arrival time. Second, there might be a disutility connected to the uncertainty of the travel time per se. This disutility can either be interpreted as an "anxiety" cost, as a "decision cost" to solve the optimal departure time problem, or as a cost for making up contingency plans for the event that a delay occurs. A third explanation may be that the travellers in this sample systematically misperceive probabilities, and thus are not really making utilitymaximizing decisions in the conventional sense. This is in contrast to other findings (e.g. de Palma el al., 2008) that travel choice tends to converge toward rational utility maximization when the trip is a familiar one. 
From a historical perspective, our findings represent a half-step backwards. Reducedform expressions, where some measure of travel time variability was introduced in an indirect utility function, were originally introduced and motivated in a rather ad-hoc manner. The theoretical underpinning of reduced-form expressions came only later, when it was shown that such models could be derived from scheduling models. Our results, however, cast some doubt on whether this derivation really captures the whole story. It seems as if the derivation of the reduced-form model from a scheduling model omits certain features essential to the understanding, analysis and valuation of travel time variability. Even if this derivation is useful for proving the microeconomic consistency of suggested reduced-form expressions, and for showing the theoretical equivalence between scheduling models and reduced-form models, this equivalence turns out not to be borne out in the empirical evidence. It seems as if the "late arrival" concept in scheduling models does not fully capture the "delay risk" central to reducedform models. Or in other words, it seems as if being "delayed" is considerably worse than just being "late".

\section{ACKNOWLEDGMENTS}

Financial support from the Swedish Transport Administration is gratefully acknowledged. Comments from two anonymous reviewers greatly improved the exposition of the paper and are gratefully acknowledged. Thanks to Robin Lindsey for valuable comments.

\section{REFERENCES}

Bates, J. (2009). An agenda for research on reliability. Proceedings of the European Transport Conference. Retrieved from http://etcproceedings.org/paper/an-agendafor-research-on-reliability

Bates, J., Black, I., Fearon, J., Gilliam, C., Porter, S. (2002). Supply models for use in modelling the variability of journey times on the highway network. Proceedings of the European Transport Conference.

Bates, J., Polak, J., Jones, P., Cook, A. (2001). The valuation of reliability for personal travel. Transportation Research Part E, 37(2-3), 191-229.

Batley, R. (2007). Marginal valuations of travel time and scheduling, and the reliability premium. Transportation Research Part E, 43(4), 387-408.

Burns, Z., Chiu, A., Wu, G. (2010). Overweighting of Small Probabilities. Encyclopedia of Operations Research and Management Science. John Wiley \& Sons.

Börjesson, M., Eliasson, J. (2011). On the use of "average delay" as a measure of train reliability. Transportation Research Part A, 45(3), 171-184.

de Palma, A., Ben-Akiva, M., Brownstone, D., Holt, C., Magnac, T., McFadden, D., Moffatt, P., et al. (2008). Risk, uncertainty and discrete choice models. Marketing Letters, 19(34), 269-285.

Fosgerau, M., Engelson, L. (2011). The value of travel time variance. Transportation Research Part B, 45(1), 1-8. 
Fosgerau, M., Karlström, A. (2010). The value of reliability. Transportation Research Part B, 44(1), 38-49.

Hakes, J. K., Viscusi, W. K. (2004). Dead Reckoning: Demographic Determinants of the Accuracy of Mortality Risk Perceptions. Risk Analysis, 24(3), 651-664.

Hjorth, K. (2010). Notes on VOR model for discrete travel time distribution. Technical Note, DTU Transport, Copenhagen.

Jenelius, E., Mattsson, L.-G., Levinson, D. (2011). Traveler delay costs and value of time with trip chains, flexible activity scheduling and information. Transportation Research Part B, 45(5), 789-807.

Lam, T. C., Small, K. A. (2001). The value of time and reliability: measurement from a value pricing experiment. Transportation Research Part E, 37(2-3), 231-251.

Lichtenstein, S., Slovic, P., Fischhoff, B., Layman, M., Combs, B. (1978). Judged frequency of lethal events. Journal of Experimental Psychology: Human Learning and Memory, 4(6), 551-578.

Morgan, M. G., others. (1983). On judging the frequency of lethal events: A replication. Risk Analysis, 3(1), 11-16.

Noland, R. B., Small, K. A., Koskenoja, P. M., Chu, X. (1998). Simulating travel reliability. Regional Science and Urban Economics, 28(5), 535-564.

Small, K. A. (1982). The Scheduling of Consumer Activities: Work Trips. The American Economic Review, 72(3), 467-479.

Snickars, F., Weibull, J. W. (1977). A minimum information principle: Theory and practice. Regional Science and Urban Economics, 7(1-2), 137-168.

Tseng, Y.-Y., Verhoef, E. T. (2008). Value of time by time of day: A stated-preference study. Transportation Research Part B, 42(7-8), 607-618.

Vickrey, W. (1973). Pricing, metering, and efficiently using urban transportation facilities. Highway Research Record, 476, 36-48.

Vickrey, W. S. (1969). Congestion Theory and Transport Investment. The American Economic Review, 59(2), 251-260.

Wilson, A. G. (1967). A statistical theory of spatial distribution models. Transportation Research, 1(3), 253-269.

Wilson, A. G. (1970). Entropy in Urban and Regional Modelling. Pion Press, London, UK. 


\section{APPENDIX A}

We derive the reduced-form expression in the step model, assuming a binary travel time distribution, as follows. First, assume that the travel time is $t$ with probability $(1-p)$, and $t+L$ with probability $p$. The reduced form expression corresponding to the "step" scheduling model can be derived directly, or using Fosgerau and Karlström's (2010) general formula. An application of Fosgerau and Karlström's approach to a discrete travel time distribution has been demonstrated by Hjorth (2010).

To derive the reduced-form expression directly, note first that if a traveller with a preferred arrival time at time zero departs at time $d$, she will get an expected utility of:

$E[u(d, a)]=E[-\alpha(a-d)+\beta \min (0, a)-\gamma \max (0, a)]=$ $=-\alpha(t+p L)+\beta\left[p(t+L+d)^{-}+(1-p)(t+d)^{-}\right]-\gamma\left[p(t+L+d)^{+}+(1-\right.$ $\left.p)(t+d)^{+}\right]$,

where the parameters $\alpha, \beta$ and $\gamma$ are all positive, and where the superscripts $x^{-}\left(x^{+}\right)$ denote only the negative (positive) cases of $x$, otherwise the quantity is set to zero. Let $d^{*}$ be the optimal departure time and $u^{*}$ be the maximal achieved utility, i.e. $d^{*}=\operatorname{argmax}(E U)$ and $u^{*}=\max (E U)$. Given that $\frac{\beta}{\beta+\gamma}<1$, i.e. that it is worse to arrive late than to arrive early, we always have $d^{*}+t \leq 0$, otherwise the traveller is always late. This implies that the last term is always zero. Moreover, we always have $d^{*}+t+L \geq 0$, since $\beta>0$ and $\gamma>0$. This means that we can rewrite the expression as:

$E[u]=-\alpha(t+p L)+\beta(1-p)(t+d)-\gamma p(t+L+d),-t-L \leq d \leq-t$

Depending on whether $\beta / \gamma$ is greater or less than $p /(1-p)$, we get one of two cases:

$\begin{array}{cccc}\frac{\beta}{\gamma}>\frac{p}{1-p}: & d^{*}=-t, & u^{*}=-\alpha t-(\gamma+\alpha) p L & \text { (Case I) } \\ \frac{\beta}{\gamma}<\frac{p}{1-p}: & d^{*}=-t-L, & u^{*}=-\alpha t-[\beta+p(\alpha-\beta)] L & \text { (Case II) }\end{array}$

In case I, the expected lateness penalty $(\not p)$ is small, so the traveller will still depart just to be in time if no delay occurs. In case II, the expected lateness penalty is so large that the traveller starts early enough to always be on time.

The same expression can be derived using the general Fosgerau-Karlström formula. Let $\mu$ be the mean travel time and $\sigma$ be the standard deviation of the travel time. For the case of a binary travel time, we have:

$T=\left\{\begin{array}{cc}t & \text { with probability } 1-p \\ t+L & \text { with probability } p\end{array}\right.$

$\mu=t(1-p)+(t+L) p=t+p L$

$\sigma=L \sqrt{p(1-p)}$

To express the reduced-form utility in terms of scheduling parameters, we use the approach presented by Fosgerau and Karlström (2010). We start by taking the standardized distribution of travel times: 
$X=\frac{T-\mu}{\sigma}$

$X=\left\{\begin{array}{lc}x_{1} & \text { for } 1-p \\ x_{2} & \text { for } p\end{array}\right.$

where:

$x_{1} \equiv \frac{t-\mu}{\sigma}=\frac{t-t(1-p)-(t+L) p}{\sigma}=-\frac{p L}{\sigma}$

$x_{2} \equiv \frac{t+L-\mu}{\sigma}=\frac{t+L-t(1-p)-(t+L) p}{\sigma}=\frac{(1-p) L}{\sigma}$

Note that we leave $a$ and $b$ in terms of $\sigma$, since the standard deviation will cancel out at later stages. (The same derivation can be made without standardizing the travel time distribution by $\sigma$, but we leave it in here for consistency with earlier literature.)

Now, define the cumulative distribution of the standardized travel time distribution as $\Phi(x)$. With the binary distribution given above, this distribution follows a step function:

$\Phi(x)=\left\{\begin{array}{cc}0 & \text { for } x<x_{1} \\ 1-p & \text { for } x_{1} \leq x<x_{2} \\ 1 & \text { for } x \geq x_{2}\end{array}\right.$

We invert this to find the quantile function, $\Phi^{-1}(r)$ :

$\Phi^{-1}(r)= \begin{cases}x_{1} & \text { for } 0<r \leq 1-p \\ x_{2} & \text { for } 1-p<r \leq 1\end{cases}$

In the setting used by Fosgerau and Karlström (2010), the optimal departure time, in terms of the mean and standard deviation, is given by:

$d^{*}=-\mu-\sigma * \Phi^{-1}\left(1-\frac{\beta}{\beta+\gamma}\right)$

Because of the discontinuous nature of the quantile function $\Phi^{-1}(r)$, we can define its value in terms of where in the range $0<1-p<1$ lies the quantity $1-\frac{\beta}{\beta+\gamma}$ using this to find the optimal departure time depending on $\frac{\beta}{\beta+\gamma}$ :

Case I

$$
\begin{aligned}
& 1-\frac{\beta}{\beta+\gamma}<1-p \rightarrow \frac{\beta}{\beta+\gamma}>p \rightarrow \Phi^{-1}\left(1-\frac{\beta}{\beta+\gamma}\right)=x_{1} \\
& d^{*}=-\mu-\sigma x_{1}=p L-t-p L=-t
\end{aligned}
$$

Case II

$$
\begin{aligned}
& 1-\frac{\beta}{\beta+\gamma}>1-p \rightarrow \frac{\beta}{\beta+\gamma}<p \rightarrow \Phi^{-1}\left(1-\frac{\beta}{\beta+\gamma}\right)=x_{2} \\
& d^{*}=-\mu-\sigma x_{1}=-t-p L-(1-p) L=-t-L
\end{aligned}
$$


As a consequence of these optimal departure times with respect to the two cases, we can compute disutility using the formula for mean lateness given by Fosgerau and Karlström (2010):

$H\left(\Phi, \frac{\beta}{\beta+\gamma}\right)=\int_{1-\frac{\beta}{\beta+\gamma}}^{1} \Phi^{-1}(s) d s$

$u^{*}=-\alpha \mu-(\beta+\gamma) H\left(\Phi, \frac{\beta}{\beta+\gamma}\right) \sigma$

Under Case I, where a risk-taking traveller chooses a later departure and risks being delayed, we have:

$H\left(\Phi, \frac{\beta}{\beta+\gamma}\right)=x_{1}\left(\frac{\beta}{\beta+\gamma}-p\right)+x_{2} p=-\frac{p L}{\sigma}\left(\frac{\beta}{\beta+\gamma}-p\right)+\frac{(1-p) L}{\sigma} p=\frac{p L}{\sigma}\left(1-\frac{\beta}{\beta+\gamma}\right)$

$u^{*}=-\alpha \mu-(\beta+\gamma) H\left(\Phi, \frac{\beta}{\beta+\gamma}\right) \sigma=-\alpha(t+p L)-(\beta+\gamma) \frac{p L}{\sigma}\left(1-\frac{\beta}{\beta+\gamma}\right) \sigma=-\alpha t-$

$(\alpha+\gamma) p L$

The first term has the scheduled travel time weighted by a value of time $\alpha$, and the second term has expected delay weighted by the value of late delays $(\alpha+\gamma)$.

Under Case II, where a risk-averse traveller chooses an earlier departure time and avoids the possibility of delay, we have:

$H\left(\Phi, \frac{\beta}{\beta+\gamma}\right)=x_{2} \frac{\beta}{\beta+\gamma}=\frac{(1-p) L}{\sigma} \frac{\beta}{\beta+\gamma}$

$u^{*}=-\alpha \mu-(\beta+\gamma) H\left(\Phi, \frac{\beta}{\beta+\gamma}\right) \sigma=-\alpha(t+p L)-(\beta+\gamma) \frac{(1-p) L}{\sigma} \frac{\beta}{\beta+\gamma} \sigma=-\alpha t-[\beta+$

$(\alpha-\beta) p] L$

That is, the first term for the scheduled travel time weighted by $\alpha$, and the second term for the delay has mixed weights, where the cost of the certain headway is fully $\beta$, but the cost of extra travel time, $p(\alpha-\beta)$, is contingent on the delay actually occurring (which has probability $p$ ).

\section{APPENDIX B}

We will derive the difference in utility in the slope scheduling model between an optimal trip with travel time $T$ and an alternative trip which deviates from the optimal trip in that the departure time is shifted by $\Delta d$ and the arrival time is shifted by $\Delta a$.

First, the utility of the optimal trip (given travel time $T$ ) is given in (13):

$u^{*}=-\beta_{0} T-\frac{\gamma_{1}}{2}\left(d^{*}+T\right)^{2}+\frac{\beta_{1}}{2}\left(d^{*}\right)^{2}$

Assume now that departure and arrival time changes relative to the optimal trip. The departure time deviation is $\Delta d$ from $d^{*}$ and the arrival time deviation is $\Delta a$ from the optimal arrival time $d^{*}+T$, such that the deviation in the travel time is $\Delta T=\Delta a-\Delta d$. The utility of this trip is then:

$u^{\mathrm{dev}}=-\beta_{0}(T+\Delta a-\Delta d)-\frac{\gamma_{1}}{2}\left(d^{*}+T+\Delta a\right)^{2}+\frac{\beta_{1}}{2}\left(d^{*}+\Delta d\right)^{2}$ 
The difference in utility due to these changes in trip schedule, compared to the optimal trip, is then $\Delta u=u^{\text {dev }}-u^{*}$ :

$\Delta u=-\beta_{0}(\Delta a-\Delta d)-\frac{\gamma_{1}}{2}(\Delta a)^{2}+\frac{\beta_{1}}{2}(\Delta d)^{2}-\gamma_{1}\left(d^{*}+T\right) \Delta a+\beta_{1} d^{*} \Delta d$

Using (11) we have that:

$$
\begin{aligned}
& \Delta u=-\beta_{0}(\Delta a-\Delta d)-\frac{\gamma_{1}}{2}(\Delta a)^{2}+\frac{\beta_{1}}{2}(\Delta d)^{2}-\gamma_{1}\left(\frac{\gamma_{1}}{\beta_{1}-\gamma_{1}} T+T\right) \Delta a+\beta_{1} \frac{\gamma_{1}}{\beta_{1}-\gamma_{1}} T \Delta d \\
= & -\beta_{0}(\Delta a-\Delta d)-\frac{\gamma_{1}}{2}(\Delta a)^{2}+\frac{\beta_{1}}{2}(\Delta d)^{2}-\frac{\gamma_{1} \beta_{1}}{\beta_{1}-\gamma_{1}} T \Delta a+\frac{\gamma_{1} \beta_{1}}{\beta_{1}-\gamma_{1}} T \Delta d \\
= & -\left(\beta_{0}+\frac{\gamma_{1} \beta_{1}}{\beta_{1}-\gamma_{1}} T\right)(\Delta a-\Delta d)-\frac{\gamma_{1}}{2}(\Delta a)^{2}+\frac{\beta_{1}}{2}(\Delta d)^{2}
\end{aligned}
$$

